\title{
MECANISMOS DE COAGULOPATIA NO TRANSPLANTE DE FÍGADO
}

\author{
Mechanisms of coagulopathy in liver transplantation
}

\author{
David Silveira Marinho ${ }^{1,2}$, Cláudia Regina Fernandes³, Joel Avancini Rocha Filho", José Otávio Costa Auler Júnior , \\ José Carlos Rodrigues Nascimento ${ }^{1,2}$
}

\begin{abstract}
RESUMO
Pacientes cirróticos costumam apresentar defeitos em múltiplos componentes da hemostasia e cuja intensidade costuma acompanhar o agravamento da insuficiência hepática. Nesse sentido, alterações nas hemostasias primária e secundária e na fibrinólise podem estar presentes. Para alcançar a cura dessa doença, entretanto, esses pacientes precisam passar pelo sério desafio à coagulação representado pelo transplante hepático, mesmo com a fragilidade previamente descrita. Durante essa cirurgia, há extensa dissecção tecidual, cujo dano pode ser agravado por hipertensão portal e aderências. Além disso, é comum a ocorrência de um ambiente desfavorável à coagulação, com acidose, hipocalcemia e hipotermia. Reunidos, esses e outros fatores tornam frequente a ocorrência de estados de coagulopatia e demanda por medidas de suporte à hemostasia. O conhecimento das causas e dos mecanismos envolvidos no problema confere maior precisão às medidas terapêuticas escolhidas.
\end{abstract}

Descritores: Cirrose hepática; Transplante de Fígado; Coagulação Sanguínea; Transfusão Sanguínea.

Instituições:

${ }^{1}$ Unidade de Transplante Hepático do Hospital Geral de Fortaleza HGF, Fortaleza/CE, Brasil.

2 Serviço de Anestesiologia do Hospital Geral de Fortaleza - HGF, Fortaleza/CE, Brasil.

${ }^{3}$ Serviço de Anestesiologia do Hospital Universitário Walter Cantídio da Faculdade de Medicina da Universidade Federal do Ceará, Fortaleza/CE, Brazil.

${ }^{4}$ Disciplina de Anestesiologia do Hospital das Clínicas da Faculdade de Medicina da Universidade de São Paulo, São Paulo/SP, Brazil.

Correspondência:

David Silveira Marinho

Rua Afonso Celso, 196, Apt.1202, bloco 1, Fortaleza/CE.

Tel.: (85) 99174-6188.

E-mail: davidsmarinho@gmail.com

Recebido em: 14/01//2018

Aceito em: 09/02/2018

\section{INTRODUÇÃO}

Durante a cirurgia de transplante ortotópico de fígado (TOF), não bastassem as múltiplas anormalidades basais no perfil hemostático dos cirróticos, outras ameaças à coagulação são impostas. ${ }^{1}$ Além do comprometimento da hemostasia, dificuldades cirúrgicas também influenciam a magnitude do sangramento, como a vasta proliferação de circulação colateral, o ingurgitamento venoso decorrente da hipertensão portal e a eventual presença de aderências decorrentes de cirurgias prévias. ${ }^{2}$ Por essas razões, grandes sangramentos têm sido comuns desde os primeiros transplantes hepáticos até a atualidade, e ainda constituem um grande desafio no manejo perioperatório. Esta revisão acadêmica tentou compilar e descrever os principais mecanismos de coagulopatia no transplante de fígado, permitindo maior precisão em seu tratamento. 


\section{MÉTODO}

Trata-se de revisão integrativa da literatura por meio de busca nas bases eletrônicas de dados Pubmed e Periódicos CAPES. Os descritores em inglês utilizados foram: "machine perfusion" e "liver preservation". Foram selecionados 73 trabalhos, incluindo artigos de revisão, estudos experimentais, além de estudos clínicos em humanos. Apenas trabalhos de língua inglesa foram incluídos.

\section{PAPEL DO FÍGADO NA HEMOSTASIA}

No final do século XIX e início do século $X X$, foram publicados vários estudos sobre a fisiologia hepática. Muitos destes avaliaram o impacto que o comprometimento da função hepática teria sobre a coagulação. Naquela época, para induzir o dano hepático, utilizou-se a intoxicação, ${ }^{3}$ a hepatectomia ${ }^{4}$ ou a simples exclusão vascular do fígado. ${ }^{5} \mathrm{~A}$ partir daí, percebeuse o importante papel do fígado na coagulação, e então, especulou-se que o fígado seria o principal sítio de síntese dos fatores da coagulação, fato que depois foi comprovado experimentalmente. ${ }^{6}$

$\mathrm{Na}$ hemostasia primária, o fígado atua como o principal produtor de trombopoietina (TPO), a citocina primordial na maturação dos megacariócitos e na formação de plaquetas.

Esse órgão também ocupa importante papel na hemostasia secundária, ao ser o principal sítio da síntese de fibrinogênio e dos demais fatores pró-coagulantes (II, V, VII, VIII, IX, X, XI, XII, XIII). Os fatores VIII e XIII também possuem sítios extrahepáticos de síntese, mas a quantidade produzida geralmente é bem inferior à sintetizada no fígado. Além dos fatores prócoagulantes, esse órgão também tem função importante na produção das principais proteínas anticoagulantes, como Proteína C, Proteína S e Antitrombina III.

Fisiologicamente, em paralelo ao processo hemostático, existe um mecanismo fisiológico de proteção contra a trombose: a fibrinólise. Esta é considerada a contraparte do sistema da coagulação, já que ela age continuamente prevenindo a deposição excessiva de fibrina nas paredes dos vasos. Todas as proteínas envolvidas na fibrinólise, exceto o Ativador Tecidual do Plasminogênio (tPA) e o Inibidor 1 do Ativador do Plasminogênio (PAl-1), são sintetizadas pelo fígado.

\section{A HEMOSTASIA NO PACIENTE CIRRÓTICO}

Pelo fato de o fígado desempenhar papel tão central no sistema hemostático, o comprometimento da função hepatocelular na cirrose hepática pode resultar em anormalidades que se estendem além da síntese dos fatores pró-coagulantes nos hepatócitos. A deposição de fibrina, que representa o passo final da coagulação, depende do delicado balanço entre ativação, inibição e desintegração dos fatores da coagulação.
$\mathrm{Na}$ hepatopatia avançada, alterações em cada um desses componentes hemostáticos têm sido demonstradas.

\subsection{Fatores pró-coagulantes e anticoagulantes}

Como o fígado é o principal sítio de síntese dos fatores da coagulação, é esperado que os níveis séricos dessas proteínas diminuam à medida que se agrava e se estende o dano hepatocelular decorrente da cirrose. Os primeiros fatores a cair são aqueles com menor meia-vida, como os fatores $\mathrm{V}$ (12 horas) e VII (4 a 6 horas). Por esse motivo, o nível plasmático do fator VII foi avaliado como marcador prognóstico na insuficiência hepática aguda. ${ }^{7}$ A concentração sérica do fator VIII, entretanto, não se correlaciona com o grau de disfunção hepática. Seus níveis, na verdade, costumam estar elevados em doentes hepatopatas. ${ }^{8}$ Dentre os fatores que podem contribuir para esse achado estão a existência de sítios de síntese extra-hepática (como o endotélio vascular) e a elevação dos níveis de Fator de von Willebrand, que estabilizam o fator VIII circulante. ${ }^{9}$ As proteínas anticoagulantes, como Proteína C, Proteína $S$ e Antitrombina III também são reduzidas na cirrose hepática.

A vitamina $\mathrm{K}$ também parece exercer papel importante na disfunção hemostática dos pacientes cirróticos. A deficiência dessa vitamina está presente em 2 a 23\% dos pacientes hepatopatas. As possíveis etiologias são a carência nutricional, especialmente na hepatopatia alcoólica e a redução na produção ou na liberação de bile para os intestinos, já que os sais biliares são essenciais na sua absorção. Por mediar a carboxilação de resíduos de glutamato, a gama-carboxiglutamato na região $\mathrm{N}$-terminal, a vitamina $\mathrm{K}$ é fundamental para ativar os fatores II, VII, IX, X, e as proteínas $\mathrm{C}$ e $\mathrm{S}$. As proteínas que não passam por essa modificação estrutural não permitem a ligação de íons cálcio e não conseguem desempenhar sua função na coagulação. ${ }^{10}$

\subsection{Fibrinogênio}

Por ser uma proteína de fase aguda e possuir sítios de síntese extra-hepática, o fibrinogênio costuma ter níveis plasmáticos normais ou elevados em pacientes com doença hepática leve a moderada. ${ }^{11}$ Níveis reduzidos são mais comumente observados quando há grave comprometimento da função hepatocelular, na hepatite fulminante ou em casos complicados por hiperfibrinólise, coagulação intravascular disseminada e sangramentos maciços. ${ }^{12}$ Além das alterações quantitativas do fibrinogênio, 60 a $70 \%$ dos pacientes cirróticos apresentam alterações qualitativas do fibrinogênio. ${ }^{13}$ A disfibrinogenemia da hepatopatia crônica terminal é decorrente de um aumento na atividade da enzima sialil-transferase nos hepatócitos em regeneração, resultando em aumento nos resíduos de ácido siálico nas moléculas de fibrinogênio produzidas. ${ }^{14}$ Como o ácido siálico é carregado negativamente, as moléculas de fibrinogênio com excesso desse carboidrato repelem-se, o 
que retarda ou compromete a polimerização da fibrina. Além disso, as anomalias estruturais no fibrinogênio e na fibrina tornam-nos mais suscetíveis à fibrinólise.

\subsection{Plaquetas}

Em cirróticos, a despeito da capacidade de a medula óssea aumentar a produção de plaquetas, a contagem destas pode estar reduzida ou normal. Entretanto, a trombocitopenia tende a ser o achado mais prevalente, sendo encontrada em 49 a $64 \%$ desses pacientes. ${ }^{15}$ A etiologia da trombocitopenia é multifatorial. Como a esplenomegalia é um clássico achado da hipertensão portal, o sequestro esplênico de plaquetas foi, historicamente, considerado como o principal fator etiológico. Entretanto, atualmente, entende-se que a redução na produção de TPO, causada pela perda de parênquima hepático funcionante é a principal causa da trombocitopenia associada à cirrose. ${ }^{16}$ Além disso, níveis elevados de anticorpos IgG contra plaquetas estão elevados em pacientes cirróticos, sugerindo algum papel da destruição imuno-mediada de plaquetas. $^{17}$ Outras causas, como deficiências nutricionais (folato, cobalamina), álcool, sepse, coagulação intravascular disseminada (CIVD) e drogas também podem contribuir em casos individuais. ${ }^{18}$

Paralelamente aos déficits quantitativos, defeitos qualitativos das plaquetas também estão presentes em hepatopatas. A existência de um defeito plaquetário funcional já é conhecida há algum tempo. A agregometria plaquetária demonstrou clara hipoagregabilidade em resposta à estimulação com colágeno, trombina, ácido araquidônico, Difosfato de Adenosina (ADP), adrenalina e ristocetina. ${ }^{19} \mathrm{~A}$ função plaquetária anormal tem sido atribuída ao excesso de inibidores plaquetários na circulação, como produtos da degradação de fibrina, D-dímeros e óxido nítrico. ${ }^{18}$ Defeitos plaquetários intrínsecos também têm sido relatados, como a deficiência de receptores GP1B, defeitos na transdução do sinal, síntese reduzida de tromboxano-A2 e alteração na composição da membrana fosfolipídica plaquetária. ${ }^{20}$ Como nenhum dos defeitos acima ocorre consistentemente, acredita-se que a disfunção plaquetária seja multifatorial, envolvendo fatores intrínsecos e extrínsecos.

\subsection{Hiperfibrinólise}

A associação entre doença hepática e hiperfibrinólise foi inicialmente, descrita há quase um século, quando se percebeu a rápida reliquidificação de sangue coagulado de pacientes cirróticos. ${ }^{21}$ Várias anormalidades laboratoriais relativas à fibrinólise estão descritas na cirrose. Os níveis de tPA estão aumentados, provavelmente por redução da depuração hepática e consequente aumento na sua meia-vida; já a concentração de PAI-1 é elevada na hepatopatia crônica, mas reduzida na doença hepática grave. De maneira oposta, os níveis de plasminogênio, alfa-2-antiplasmina e o Inibidor da Fibrinólise Ativável por Trombina (TAFI) estão reduzidos na doença hepática. O fator XIII, que viabiliza formação de ligações cruzadas entre os polímeros de fibrina para torná-los mais resistentes à fibrinólise também está reduzido na cirrose hepática. ${ }^{22}$

De posse desses dados laboratoriais, comumente entende-se que a cirrose está associada à hiperfibrinólise, devido (a) à redução na depuração hepática dos ativadores da plasmina e (b) à redução na síntese hepática dos principais inibidores da plasmina (Figura 1). Estudos comparando a hemostasia em transplantes hepáticos ortotópicos e heterotópicos foram especialmente úteis para validar este entendimento. ${ }^{23}$ Por estes motivos, o balanço entre esses componentes pesa no sentido da hiperfibrinólise à medida que se agrava a hepatopatia, estando ela presente em cerca de $30 \%$ dos pacientes cirróticos.

Embora seja intuitivo conceber a presença de hiperfibrinólise na cirrose, alguns autores questionam sua prevalência e relevância clínica. É possível que ela seja superestimada quando se avaliam apenas os marcadores de fibrinólise sem medir propriamente a lise do coágulo. ${ }^{24}$ Isso reflete a carência crônica de métodos para a avaliação da fibrinólise que havia até a disseminação de testes que avaliam as propriedades viscoelásticas do coágulo, considerados, atualmente, o padrão-ouro para o diagnóstico de hiperfibrinólise. Um estudo usando essa avaliação em 84 pacientes com cirrose descompensada não conseguiu detectar evidência de hiperfibrinólise. ${ }^{25}$ Isso pode sinalizar que, aparentemente, a maior parte da atenção acadêmica foi direcionada para os componentes individuais da fibrinólise, em vez de investigar a atividade fibrinolítica global.

Eventualmente, as anormalidades laboratoriais da cirrose descompensada relembram aquelas da CIVD, tais como prolongamento do Tempo de Protrombina (TP) e do Tempo de Tromboplastina Parcial Ativada (TTPa), hipofibrinogenemia, aumento nos produtos de degradação da fibrina e trombocitopenia. Entretanto, níveis plaquetários relativamente estáveis, níveis de fator VIII elevados e ausência de geração descompensada de trombina distinguem o processo que ocorre na cirrose daquele existente na CIVD. ${ }^{26}$ Além disso, lesões orgânicas causadas pela coagulação intravascular não ocorrem habitualmente, embora microtromboses possam estar presentes em certos pacientes.

A fim de contornar esses debates e caracterizar um processo único e peculiar aos pacientes cirróticos descompensados, propôs-se o termo "Coagulação Intravascular Acelerada e Hiperfibrinólise” (AICF). ${ }^{27}$ A AICF é resultado da formação de um coágulo de fibrina que é mais suscetível à degradação de plasmina devido (a) aos altos níveis de tPA combinados com a liberação inadequada de PAl-1 para controlar o tPA, (b) à presença de moléculas disfuncionais de fibrinogênio e (c) à falta de alfa-2-antiplasmina para combater a atividade da 
Mecanismos de coagulopatia no transplante de fígado

Figura 1 - Ativação e inibição da fibrinólise

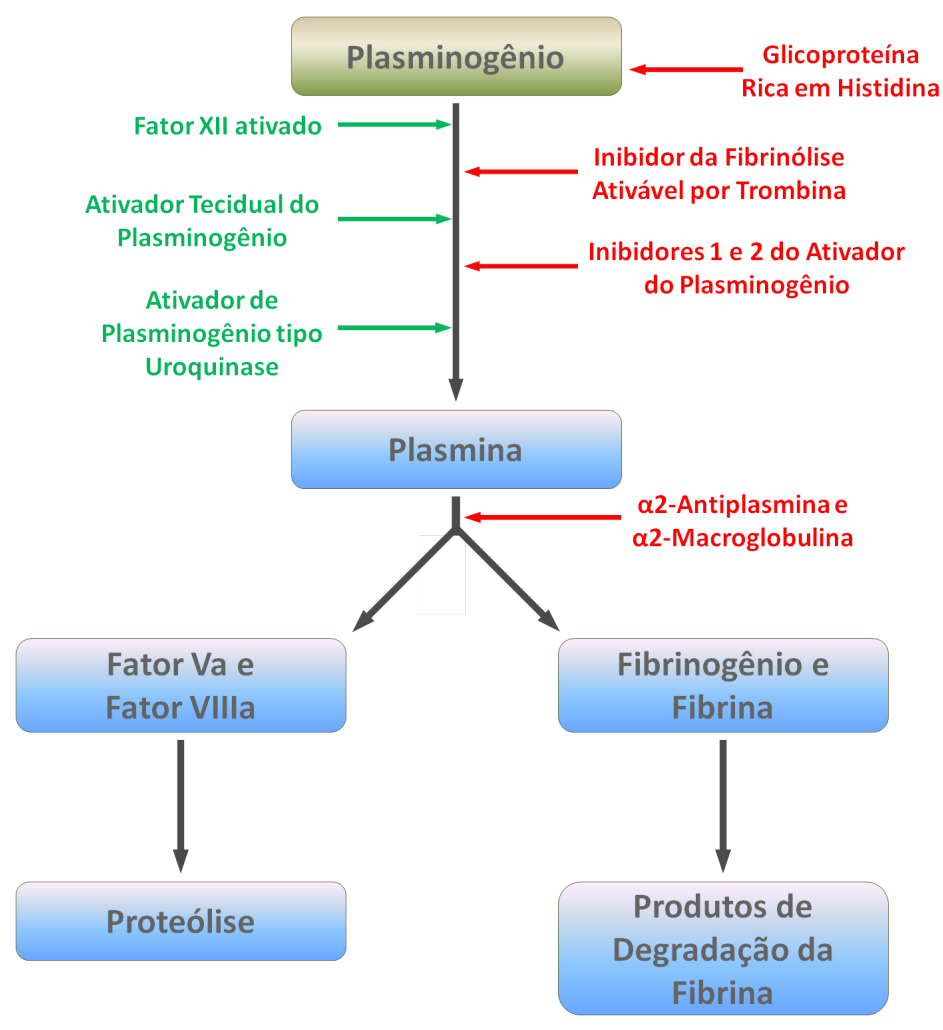

Em verde e vermelho, estão os ativadores e os inibidores da fibrinólise, respectivamente. XIla, Va e VIIla representam as formas ativadas dos fatores XII, V e VIII, respectivamente.

plasmina. ${ }^{24}$ Demonstrou-se essa nova entidade patológica em cerca de $30 \%$ dos cirróticos, mantendo relação com o grau de acometimento hepático. ${ }^{26}$

Estresses fisiológicos, como infecção, cirurgia e sangramento ou a simples presença de shunts porto-sistêmicos, por aumentar a liberação de tPA, podem agravar os desequilíbrios que desencadeiam hiperfibrinólise e a AICF2. As propriedades fibrinolíticas do líquido ascítico também têm sido implicadas na gênese dessas alterações. ${ }^{28}$

\subsection{Mecanismos fisiopatológicos adicionais}

Além das múltiplas modificações no sistema hemostático descritas anteriormente, os pacientes cirróticos podem apresentar outras anormalidades que causam prejuízos à coagulação.

\subsubsection{Infecção, endotoxemia e heparinoides endógenos}

Infecções bacterianas são comuns em pacientes cirróticos, especialmente naqueles com sangramento gastrintestinal. Além disso, peritonite bacteriana comumente precede sangramento por varizes e estudos recentes demonstraram que profilaxia antimicrobiana previne ressangramentos precoces. ${ }^{29}$ Estas observações ilustram o relevante papel etiológico das infecções no sangramento por varizes.

Endotoxemia é um achado comum em pacientes cirróticos, originando-se da translocação intestinal de bactérias, mesmo quando ainda não há sinais de sepse. A sepse pode interferir na coagulação ao induzir a liberação de substâncias que inibem a agregação plaquetária, como óxido nítrico (NO) e prostaciclina, e ao produzir citocinas que levam à ativação de fatores da coagulação e fibrinólise. ${ }^{30}$ Outro mecanismo pelo qual a sepse contribui para disfunção hemostática é a liberação de fatores heparinoides. Essas substâncias são, normalmente, depuradas pelo fígado, de maneira que seus níveis estão elevados em pacientes cirróticos. Por meio de avaliação por tromboelastografia (TEG) modificada por heparinase-1 verificou-se que nenhum paciente cirrótico sem infecção apresentava atividade de heparina, enquanto que esta foi demonstrada em 28 de 30 pacientes cirróticos infectados. ${ }^{31}$ Além disso, constatou-se a presença de heparinoides endógenos em cirróticos com sangramento por varizes, o que poderia contribuir para a dificuldade em controlar o sangramento e para o ressangramento precoce. Postula-se que endotoxemia, inflamação e hipoperfusão decorrentes da infecção poderiam induzir a liberação de 
fatores heparinoides a partir do endotélio e pelos mastócitos. ${ }^{31}$ As implicações terapêuticas da presença desses fatores em pacientes cirróticos parecem merecer maior investigação.

\subsubsection{Insuficiência renal}

A insuficiência renal é um achado comum em pacientes cirróticos e, quando presente, aumenta os riscos de eventos hemorrágicos. A fisiopatologia da disfunção hemostática decorrente da insuficiência renal parece ser multifatorial.

A disfunção plaquetária tem papel preponderante e parece resultar de anormalidades plaquetárias intrínsecas e de alterações na interação das plaquetas com as paredes dos vasos. ${ }^{32}$

As toxinas urêmicas também parecem ser relevantes. Em pacientes urêmicos, há níveis elevados de ácido guanidinosuccínico, ${ }^{32}$ que promove aumento na produção de $\mathrm{NO}^{32}$ Este, por sua vez, compromete tanto a adesão plaquetária ao endotélio quanto a agregação plaquetária.

Anemia é um achado comum em pacientes urêmicos e pode comprometer a coagulação por diversos mecanismos. Estudos reológicos demonstraram que o fluxo laminar das hemácias ocorre no centro do vaso, deslocando as plaquetas para próximo da parede dos vasos e, dessa forma maximizando sua adesão e agregação à superfície endotelial lesada. ${ }^{33}$ $\mathrm{Na}$ anemia, portanto, fica comprometida a disposição das plaquetas no interior do vaso. Além disso, as hemácias podem exercer efeitos metabólicos sobre as plaquetas ao aumentar a liberação de ADP e tromboxano A2. Por fim, os eritrócitos também agem como depuradores de certos inibidores da adesão plaquetária, como NO e prostaciclina.

Ao remover várias toxinas urêmicas que prejudicam a função plaquetária, a hemodiálise reduz mas não elimina o risco de sangramento. Por outro lado, a hemodiálise habitualmente emprega anticoagulantes, além de induzir a ativação plaquetária pelo contato do sangue com superfícies artificiais. Isso causa liberação dos grânulos plaquetários, o que pode causar exaustão de sua função e favorecer sangramentos. ${ }^{34}$

\subsubsection{Disfunção endotelial}

Detectou-se que pacientes cirróticos apresentam uma disfunção endotelial, de forma que suas células endoteliais sinusoidais não respondem com vasodilatação adequada ao aumento pós-prandial do fluxo portal. ${ }^{35}$ Além do endotélio participar da hemodinâmica, ele também é parte integrante do processo hemostático, já que contribui com o tônus vasomotor e empresta sua superfície ao processo da hemostasia. Baseado nisso, tem-se especulado que essa disfunção endotelial poderia também comprometer a coagulação, por interferir na interação plaquetas-subendotélio; entretanto, maiores estudos a esse respeito são necessários. ${ }^{24}$

\subsection{Rebalanceamento da hemostasia}

Conforme minuciosamente exposto acima, a complexidade da hemostasia em pacientes cirróticos envolve múltiplas alterações que favorecem sangramento. Por outro lado, podem estar presentes algumas anormalidades que favorecem trombose, tais como (a) aumento nos níveis séricos do Fator de von Willebrand, do Fator VIII e do PAI-1 e (b) baixos níveis da proteína ADAMTS 13 (enzima que cliva o Fator de von Willebrand), das proteínas anticoagulantes C, S, Z, cofator Il da heparina, alfa-2-macroglobulina e antitrombina 3 e do Plasminogênio. $O$ entendimento atual é que essas tendências pró e anti-hemorrágicas impõem-se uma contra a outra de forma dinâmica no doente cirrótico, assemelhando-se ao comportamento de uma balança. Quando o paciente apresenta quadro clínico estável, essas forças contrabalanceiamse, sem que haja tendência a trombose ou hemorragia. Já se demonstrou nesse cenário de estabilidade clínica que o plasma de cirróticos é capaz de gerar tanta trombina quanto sujeitos hígidos. ${ }^{36}$ Entretanto, certos estímulos, como infecção bacteriana, insuficiência renal, sangramentos e cirurgias têm potencial de desequilibrar a balança para qualquer um dos lados, dependendo das circunstâncias. É igualmente importante frisar que embora o sistema de coagulação dos cirróticos apresente-se rebalanceado, ele não é tão estável quanto o de pessoas hígidas, que possuem considerável reserva funcional de substâncias pró e anticoagulantes. Desta forma, a menor quantidade de ambas essas substâncias deixa a hemostasia do paciente cirrótico mais frágil e com menor capacidade de compensar insultos que geram desequilíbrios.

\section{SANGRAMENTOS DURANTE O TRANSPLANTE DE FÍGADO (MECANISMO E IMPACTO)}

O transplante de fígado é, certamente, um dos maiores desafios à coagulação de um paciente hepatopata. Além dos múltiplos mecanismos capazes de fazer com que 0 paciente habitualmente chegue à sala de cirurgia com um déficit hemostático basal e multifatorial, somam-se também outros fatores prejudiciais à hemostasia, que são intrínsecos à execução da cirurgia.

\subsection{Dificuldades anatômicas}

O isolamento do hilo hepático e a mobilização do fígado envolvidos na técnica de preservação da veia cava exigem que se execute extensa dissecção cirúrgica no abdome superior. Os principais fatores anatômicos que aumentam a perda sanguínea durante a execução da cirurgia são a presença de aderências decorrentes de cirurgias anteriores e a extensão da circulação colateral decorrente da hipertensão portal. ${ }^{37}$

\subsection{Comprometimento dos pré-requisitos básicos para a hemostasia}


Durante o transplante hepático, as grandes perdas sanguíneas estão associadas à hipoperfusão tecidual e requerem tratamento com volumosa reposição de fluidos e, eventualmente, transfusão maciça. Por esse motivo, há uma tendência intrínseca ao procedimento para o desenvolvimento de hipotermia, acidose, hipocalcemia e hemodiluição. Todos esses fatores são pré-requisitos muito importantes para uma hemostasia funcionante. Acidose e hipotermia são fatores tão determinantes na ocorrência de coagulopatia, que esses três fatores compõem a "tríade letal", associada à alta mortalidade no trauma.

\subsubsection{Hipotermia}

A duração da operação (aproximadamente, 5-7 horas), o tamanho da incisão, a exposição dos órgãos abdominais, o bloqueio neuromuscular e a infusão de grandes quantidades de fluidos e de hemocomponentes em temperatura inferior à corporal dão origem a uma forte tendência à hipotermia durante a cirurgia. Isso é agravado quando, ao final da fase anepática, o enxerto hepático gelado $\left(4^{\circ} \mathrm{C}\right)$ é posicionado na cavidade abdominal para as anastomoses e atinge seu pior estágio logo após a reperfusão, quando grande quantidade de sangue flui por dentro do parênquima hepático. ${ }^{38}$ Independentemente dos níveis dos fatores de coagulação na vigência de hipotermia, os exames convencionais de coagulação só se apresentariam alterados caso fossem realizados na baixa temperatura apresentada pelo paciente, o que não é rotineiro. Trinta e quatro graus Celsius foi a temperatura crítica a partir da qual houve piora da coagulação a patamares perigosos. ${ }^{39}$ Até a temperatura de $35^{\circ} \mathrm{C}$, o impacto da hipotermia sobre a hemostasia é mínimo. De $35^{\circ} \mathrm{C}$ a $33^{\circ} \mathrm{C}$, a hipotermia exerce seus efeitos negativos predominantemente sobre a hemostasia primária, modificando a estrutura plaquetária durante sua adesão e reduzindo a produção e ação de ativadores plaquetários durante a sua ativação. ${ }^{40}$ Estimase que a atividade enzimática das proteases da coagulação sejam reduzidas de $4 \%$ a $10 \%$ para cada $1^{\circ} \mathrm{C}$ a menos entre $37^{\circ} \mathrm{C}$ e $33^{\circ} \mathrm{C}$, conforme também demonstrado em estudos com TEG.39 Abaixo de $33^{\circ} \mathrm{C}$, a atividade dos fatores de coagulação e a geração de trombina são reduzidas a 25$50 \%$ do normal, e seu impacto clínico passa a ser relevante. Além disso, demonstrou-se que a hipotermia inibe a síntese de fibrinogênio. ${ }^{41}$ A atividade fibrinolítica não parece ser significativamente influenciada pela hipotermia, exceto em temperaturas abaixo de $20^{\circ} \mathrm{C} .39 \mathrm{O}$ efeito global da hipotermia sobre a hemostasia é a geração de um coágulo formado lentamente e apresentando com grande fragilidade, de maneira que se torna incapaz de inibir sangramentos.

\subsubsection{Acidose}

A maioria das proteases envolvidas na coagulação atua de maneira ótima num $\mathrm{pH}$ entre 8,0 e 8,5; entretanto, cada fator de coagulação tem suscetibilidade diferente aos efeitos da acidose. ${ }^{42}$ Os mecanismos parecem envolver a interferência das altas concentrações de íons de hidrogênio nas interações iônicas entre os fatores de coagulação, superfícies fosfolipídicas e íons de cálcio. Também, se o $\mathrm{pH}$ ficar abaixo de 7,4, as plaquetas começam a mudar sua forma e a reduzir seu número. ${ }^{43}$ Quando há um valor de $\mathrm{pH}$ inferior a 7,1 ou excesso de bases superior a 12,5, ocorre (a) queda de $35 \%$ na concentração sérica de fibrinogênio por sequestro e por degradação, (b) piora na função de todos os receptores plaquetários, 42 e (c) queda de $50 \%$ nas atividades de fatores e na subsequente geração de trombina. ${ }^{44}$

\subsubsection{Hipocalcemia}

Os fatores de coagulação dependentes de vitamina K (II, VII, IX, X) e as proteínas C e S são carregados negativamente, bem como os fosfolipídios de membrana. Os íons de cálcio, que são carregados positivamente, agem como pontes entre os fatores da coagulação e os fosfolipídios do endotélio danificado. ${ }^{42} \mathrm{O}$ cálcio também exerce efeito protetor sobre a molécula de fibrinogênio, favorecendo sua polimerização em fibrina e dificultando sua proteólise pela plasmina. Esse íon também é fundamental para todas as atividades plaquetárias, para a via da proteína $\mathrm{C}$ e para a fibrinólise. Os mecanismos que favorecem a ocorrência de hipocalcemia são: quelação dos íons cálcio por ânions (especialmente lactato e citrato, este último oriundo dos hemocomponentes), quelação pela albumina utilizada como solução coloide no intraoperatório, hemodiluição dos íons de cálcio pela infusão de soluções desprovidas de cálcio e uso do cell saver, que devolve ao paciente uma suspensão de hemácias em solução salina. $\mathrm{Na}$ hipocalcemia induzida pelo citrato das transfusões, os principais determinantes são a velocidade de infusão do hemocomponente e a capacidade metabólica residual do fígado cirrótico para degradar o citrato infundido. A capacidade metabólica do fígado cirrótico, por sua vez, pode ser piorada durante a cirurgia, em decorrência de hipotermia e hipotensão comumente presentes.

\subsubsection{Hemodiluição}

Comumente, durante o TOF, as perdas sanguíneas são repostas com soluções cristaloides e/ou coloides desprovidas de fatores de coagulação, com o intuito de combater a hipovolemia. O uso dessas soluções, embora eficiente em restaurar o equilíbrio hemodinâmico, é necessariamente acompanhado da diluição de todos os componentes da coagulação, incluindo elementos celulares e proteínas pró-coagulantes, anticoagulantes, prófibrinolíticas e antifibrinolíticas.

Cada um desses componentes possui um nível crítico peculiar, abaixo do qual ele passa a ser insuficiente para manter o funcionamento da hemostasia. A depender da intensidade da hemodiluição, um ou mais dos participantes da coagulação 
poderá atingir seus níveis críticos, resultando em coagulopatia por mecanismo dilucional. O nível crítico do fibrinogênio é de $100 \mathrm{mg} / \mathrm{dL}$, sendo alcançado após uma perda aproximada de $150 \%$ do volume sanguíneo circulante; já os demais fatores de coagulação e a contagem plaquetária só atingem concentrações críticas após uma perda de $200 \%$ da volemia. ${ }^{45}$ Com base neste estudo clínico, em estudos experimentais e em modelos matemáticos, parece bem estabelecido que: (a) em cirurgias de pacientes sem outros comprometimentos da hemostasia, a coagulação só é comprometida após perdas superiores a 1,5 volemias, e (b) o fibrinogênio é o primeiro dos integrantes da coagulação a atingir níveis críticos em cenários de hemodiluição. É essencial ressaltar que essas cifras foram aferidas em indivíduos sem quaisquer anormalidades hemostáticas que se submetem a cirurgias não hepáticas. ${ }^{45}$ Dessa forma, é razoável presumir que esses indivíduos iniciaram a cirurgia com os componentes da coagulação dentro de uma concentração normal, e portanto com ampla margem de segurança contra eventuais insultos intraoperatórios à hemostasia.

Opaciente que se submete aoTOF, poroutrolado, habitualmente já inicia a cirurgia com os elementos de coagulação abaixo dos valores normais, devido ao comprometimento variável da função hepatocelular. Isso, provavelmente, Ihes confere reduzida margem de segurança contra a hemodiluição e demais insultos intraoperatórios à coagulação descritos previamente, resultando em frágil equilíbrio hemostático. Outro ponto importante é que cada paciente cirrótico exibe um padrão próprio de distúrbios hemostáticos, de maneira que em certos doentes, a trombocitopenia é a alteração mais relevante, enquanto que, em outros, a deficiência de fatores da coagulação tem papel preponderante. Também, embora haja tendência à redução global na concentração dos fatores pró e anticoagulantes nos cirróticos, não é possível correlacionar o nível de depleção desses fatores com as alterações existentes nos exames convencionais de coagulação. ${ }^{46}$ Essas peculiaridades corroboram o tênue equilíbrio hemostático do paciente cirrótico durante o TOF; entretanto, não é possível predizer a perda sanguínea tolerável de um paciente cirrótico e nem o componente da hemostasia que primeiro atingirá seus níveis críticos, desencadeando coagulopatia.

\subsection{Comportamento hemostático durante o TOF}

A cirurgia de TOF costuma ser dividida em três fases: fase de hepatectomia ou pré-anepática (desde a indução anestésica até a exclusão vascular do fígado nativo), a fase anepática e a fase neo-hepática (desde a reperfusão do enxerto até o final da cirurgia). À medida que se progride por essas fases, insultos específicos são impostos à hemostasia, alguns ao longo de toda a cirurgia (como o consumo de fatores para geração de trombina e a tendência ao descumprimento dos pré-requisitos básicos para a hemostasia) e outros mais pronunciados, em certos momentos.

\subsubsection{Fase pré-anepática}

Durante esse período inicial, a principal causa de sangramento é a lesão cirúrgica intrínseca à remoção do fígado nativo. A experiência do cirurgião, portanto, é fator determinante na perda sanguínea. A hemorragia pode ser agravada pelas alterações anatômicas descritas anteriormente, e também, pela gravidade do defeito hemostático basal do paciente. Este, por sua vez, é determinado pela etiologia e gravidade do comprometimento da função hepatocelular.

Embora com menor relevância, alguns fatores nessa primeira fase podem contribuir para uma piora da função hemostática. Além disso, a reposição das perdas sanguíneas gera necessariamente uma tendência à hemodiluição dos componentes da hemostasia, mesmo quando combina cristaloides, coloides e fatores da coagulação. ${ }^{47}$ Adicionalmente, cerca de $26 \%$ dos pacientes cirróticos submetidos a transplante hepático apresentam aumento da atividade fibrinolítica já nessa fase, por mecanismos que serão descritos na seção sobre hiperfibrinólise, a seguir. Como o fígado doente (e, eventualmente, hipoperfundido) não consegue compensar o consumo e diluição dos fatores de coagulação, é possível que os níveis críticos destes sejam alcançados, gerando, precocemente, um estado de coagulopatia.

\subsubsection{Fase anepática}

Após a exclusão vascular do fígado nativo, tem início um estado de insuficiência hepática absoluta. Nessa fase, observa-se importante queda na concentração dos fatores de coagulação. A interrupção da síntese desses fatores não parece ser o fator mais importante, visto que suas meiasvidas são superiores às habituais duas horas de duração dessa fase. Os mecanismos provavelmente mais relevantes na redução dos fatores de coagulação são o consumo para produção de coágulos, CIVD e hiperfibrinólise. ${ }^{48}$

A extensa lesão tecidual cirúrgica combina-se a um cenário de falta de depuração hepática dos ativadores de coagulação e de ausência de síntese de inibidores de coagulação. Essa combinação produz grande ativação da hemostasia e consumo de múltiplos fatores para a formação de fibrina, num estado de CIVD.

Adicionalmente a esse consumo, a fase anepática apresenta um estado hiperfibrinolítico caracterizado por aumento na geração de plasmina. Na hiperfibrinólise, essa enzima proteolítica faz a degradação seletiva de fibrina/fibrinogênio e dos fatores V e VIII, contribuindo para a redução em suas concentrações. Ainda é discutido se o estado hiperfibrinolítico do transplante hepático teria origem primária (decorrente do desequilíbrio entre ativados e inibidores da fibrinólise) ou se seria secundário à ocorrência de coagulação intravascular disseminada; ${ }^{49}$ entretanto, devido à escassez de achados histopatológicos compatíveis com CIVD nesses doentes, 
a origem primária parece mais plausível. Dependendo das demandas por fluidos e do regime de reposição volêmica empregado, a hemodiluição também pode ter papel relevante na redução dos fatores da coagulação durante a fase anepática. A presença e a relevância de cada um desses mecanismos é provavelmente variável entre diferentes pacientes.

\subsubsection{Reperfusão e seus momentos iniciais}

A reperfusão, embora constitua apenas um curto intervalo e não propriamente uma fase do TOF, causa alterações tão marcantes na hemostasia que merece ser discutida separadamente. Vários mecanismos são aventados. $\mathrm{Na}$ reperfusão, um volume considerável de fluidos que preenchem o enxerto (solução de preservação e cristaloides) é incorporado à circulação, podendo causar diluição dos fatores hemostáticos. ${ }^{50}$ Uma série de mecanismos que podem estimular a liberação endotelial de tPA estão presentes durante o TOF, especialmente na reperfusão, tais como lesão endotelial isquêmica no enxerto, uso de agentes vasopressores, acidose e estase venosa no território portal. O tPA é o principal ativador da fibrinólise e os níveis mais elevados durante o TOF são atingidos nos momentos iniciais após a revascularização. ${ }^{51}$ Além dessas quantidades maciças de tPA, a reperfusão do enxerto libera citocinas como fator de necrose tumoral e outras enzimas proteolíticas, como tripsina, elastase e catepsina $B$, que também contribuem para o estado de proteólise e coagulopatia.

Além disso, as alterações bioquímicas, fisiológicas e metabólicas da reperfusão podem influenciar negativamente a capacidade hemostática. Dessa forma, acidose metabólica, queda no desempenho cardiovascular, redução dos níveis de cálcio ionizado, redução da temperatura corporal e hipercalemia são observados na reperfusão e podem afetar negativamente o sistema de coagulação. Isso é ilustrado pelo fato de que muitas das alterações hemostáticas observadas logo após a reperfusão já melhoram após 30 minutos. Embora esse intervalo de tempo não permita a estabilização funcional do enxerto, coincide com o tempo necessário para melhora do comprometimento humoral e metabólico causado pela reperfusão. ${ }^{52}$

\subsubsection{Fase neo-hepática}

Conceitualmente, a fase neo-hepática tem início após a reperfusão do enxerto e engloba as drásticas alterações que ocorrem na reperfusão descritas no item 3.3.3. À medida que as alterações metabólicas e humorais da reperfusão vão-se dissipando vão dando espaço a outros insultos à coagulação.

A drástica ativação da coagulação que ocorre após a reperfusão do enxerto causa grande consumo dos fatores de coagulação, o que combinado com o intenso estado hiperfibrinolítico desencadeado leva os fatores de coagulação a atingir seus níveis plasmáticos mais baixos ao longo do transplante.
O sequestro de plaquetas no enxerto também pode ter algum papel nos sangramentos da fase neo-hepática. Demonstrouse que há redução de até $55 \%$ no número de plaquetas após a revascularização do enxerto hepático, e isso poderia ser causado pelo extravasamento de plaquetas para os espaços de Disse e/ou ativação do sistema reticulo-endotelial. ${ }^{53}$

O intenso estado hiperfibrinolítico desencadeado pela reperfusão se prolonga durante a fase neo-hepática. A meiavida e a atividade do tPA estão aumentadas após a reperfusão, pois a depuração do tPA e a produção de inibidores da fibrinólise são feitos pelo fígado. Esses efeitos tendem a ser tanto maiores quanto maiores forem o tamanho do enxerto e o tempo de isquemia. À medida que o enxerto vai restaurando sua função, os efeitos da liberação maciça de tPA vão-se dissipando.

A qualidade da preservação do enxerto também contribui para os distúrbios hemostáticos, pois enxertos mal preservados e/ou com tempos de isquemia prolongados podem ativar a coagulação por meio de seu endotélio lesado e/ou pela liberação de materiais com atividade tromboplástica. ${ }^{54}$

Nesta fase do TOF também pode haver circulação de fatores com efeitos hemostáticos semelhantes aos da heparina, descritos separadamente em detalhes a seguir.

\subsubsection{Liberação de fatores heparinoides}

A detecção de heparina no plasma dos receptores após a revascularização do enxerto vem sendo descrita desde os primeiros transplantes hepáticos experimentais em animais e em humanos. ${ }^{55}$ A partir daí, postulou-se que a presença de heparina ou de substâncias heparinoides poderia contribuir para a coagulopatia e para as alterações tromboelastográficas demonstradas após a reperfusão. ${ }^{52}$ Os exames convencionais de coagulação nem sempre mostram-se alterados pela presença dessas substâncias. ${ }^{31}$ Subsequentemente, com o uso da TEG modificada por heparinase-I, uma enzima que antagoniza o efeito de substâncias heparinoides sem causar alterações na TEG, passou-se a detectar essas substâncias em pacientes com exames convencionais normais. ${ }^{31}$ Tal perfil laboratorial poderia ser causado pela fração de baixo peso molecular das substâncias heparinoides endógenas.

Especula-se que as substâncias responsáveis por esse efeito heparinoide poderiam ter origem exógena ou endógena. A principal fonte de heparina exógena seria o próprio enxerto hepático, já que, atualmente, é recomendada a injeção de heparina durante a captação de órgãos, e sabe-se que ela é capaz de ligar-se a superfícies endoteliais íntegras, lesadas e até em superfícies desendotelizadas. ${ }^{56}$ Habitualmente, antes do término das anastomoses, o enxerto é irrigado com solução de Ringer com lactato e isso evacua uma fração da heparina ligada ao endotélio do enxerto. Entretanto, a fração de heparina que não foi lavada por essa irrigação será liberada na circulação do receptor, ainda mantendo sua atividade anticoagulante. ${ }^{57}$ 
Por outro lado, já se demonstrou efeito heparinoide antes da reperfusão do enxerto hepático, ${ }^{58}$ e até mesmo após a reperfusão de enxertos cujos doadores não haviam recebido heparina exógena. ${ }^{59}$ A partir dessas observações, passou-se a cogitar que substâncias endógenas com efeito heparinoide também poderiam ter algum papel nesse efeito anticoagulante.

Os heparinoides endógenos são glicosaminoglicanos que são sintetizados pela parede dos vasos e passam a fazer parte do glicocálice. Aparentemente, os mais importantes são a heparina e o heparan sulfato, mas também incluem ácido hialurônico, sulfato de condroitina, dermatan sulfato e keratan sulfato. Agem como anticoagulantes por serem cofatores da antitrombina III e ajudam a manter o balanço hemostático sobre a superfície endotelial. Episódios graves de hipoperfusão tecidual causam lesão endotelial e degradação do glicocálice, liberando essas substâncias para a circulação. ${ }^{60}$

Durante o TOF, portanto, o efeito heparinoide pode ter múltiplas origens: (a) heparina exógena presente no enxerto, cateteres preenchidos com solução heparinizada, circuito de bypass veno-venoso, soluções de irrigação utilizadas pelos cirurgiões e sangue recuperado com uso de cell saver (especialmente quando se utiliza o programa de lavagem de emergência); (b) heparinoides endógenos oriundos de lesão endotelial durante a isquemia do enxerto; e (c) heparinoides endógenos produzidos pelo endotélio do receptor durante episódios graves de hipoperfusão tecidual (comuns durante a primeira fase da cirurgia do TOF). As alterações tromboelastográficas foram mais acentuadas após a revascularização do enxerto, ${ }^{58}$ dando a entender que essa pode ser a principal fonte de substâncias heparinoides. Demonstrou-se que quanto maior o comprometimento da atividade hepatocelular, maior a atividade de heparina, ${ }^{58}$ o que pode ser explicado pelo fato de a depuração das substâncias heparinoides exógenas e endógenas ser dependente de função hepática adequada.

Embora sua presença e seu efeito anticoagulante sejam incontestes, a quantificação da sua contribuição para a ocorrência de sangramentos nesses pacientes e a necessidade de tratamento ainda merecem mais estudos. ${ }^{31}$

\section{CONCLUSÃO}

A hemostasia no paciente cirrótico apresenta anormalidades em múltiplos de seus componentes. Por sua vez, o transplante hepático é uma cirurgia de grande porte e cujo curso envolve, necessariamente, comprometimento e cessação da função hepática residual seguidos por um retardo até a restauração desta função no enxerto recém-implantado. Um conhecimento amplo a respeito dos possíveis déficits e de seus mecanismos é fundamental para instituir o tratamento adequado.

\section{ABSTRACT}

Cirrhotic patients usually present failures in multiple hemostasis components and whose intensity usually follows the aggravation of hepatic insufficiency. In this sense, changes in primary and secondary hemostasis and fibrinolysis may be present. However, to achieve cure for this disease, those patients need undergo the serious challenge of coagulation represented by liver transplantation, even with the previously described fragility. During this surgery, there is an extensive tissue dissection, whose damage can be aggravated by portal hypertension and adhesions. In addition, an unfavorable environment to coagulation, that is, with acidosis, hypocalcemia and hypothermia is common. Together, these and other factors frequently lead to the occurrence of coagulopathy and a demand for hemostasis support measures. Knowing the causes and mechanisms involved in the problem provides greater accuracy to the therapeutic measures chosen.

Keywords: Hepatic Cirrhosis; Liver Transplantation; Blood Clotting; Blood Transfusion. 


\section{REFERÊNCIAS}

1. Forkin KT, Colquhoun DA, Nemergut EC, Huffmyer JL. The Coagulation Profile of End-Stage Liver Disease and Considerations for Intraoperative Management. Anesth Analg. 2018;126(1):46-61.

2. Senzolo M, Burra P, Cholongitas E, Burroughs AK. New insights into the coagulopathy of liver disease and liver transplantation. World J Gastroenterol. 2006;12(48):7725-36.

3. Doyon M. Incoagulabilité du sang provoquée par le chloroforme; rôle du foie. Comptes rendus hebdomadaires des seances et memoires de la Societe de biologie. 1905;57:30-1.

4. Jones TB, Smith HP. The blood fibrinogen level in hepatectomized dogs and an outline of a method for the quantitative determination of fibrinogen. Am J Physiol. 1930;94(1):144-61.

5. Doyon M, Gautier C. Phénomènes tétaniques provoqués par l'anémie artérielle du foie. Comptes rendus hebdomadaires des seances et memoires de la Societe de biologie. 1907;62:429-30.

6. Miller LL, Bale WF. Synthesis of all plasma protein fractions except gamma globulins by the liver; the use of zone electrophoresis and lysine-epsilon-C14 to define the plasma proteins synthesized by the isolated perfused liver. J Exp Med. 1954;99(2):125-32.

7. Dymock IW, Tucker JS, Woolf IL, Poller L, Thomson JM. Coagulation studies as a prognostic index in acute liver failure. Br J Haematol. 1975;29(3):385-95.

8. Eby CS, Joist JH. Hemostatic abnormalities in liver disease. In: Colman RW, Marder VJ, Clowes AW, George JN, Goldhaber SZ, editors. Hemostasis and Thrombosis: Basic Principles and Clinical Practice. 5th ed. Philadelphia, PA: Lippincott Williams \& Wilkins; 2006. p. 1025-33.

9. Hollestelle MJ, Geertzen HG, Straatsburg IH, van Gulik TM, van Mourik JA. Factor VIII expression in liver disease. Thromb Haemost. 2004;91(2):267-75.

10.Blanchard RA, Furie BC, Jorgensen M, Kruger SF, Furie B. Acquired vitamin K-dependent carboxylation deficiency in liver disease. N Engl J Med. 1981;305(5):242-8.

11.Tytgat GN, Collen D, Verstraete M. Metabolism of fibrinogen in cirrhosis of the liver. J Clin Invest. 1971;50(8):1690-701.

12.Violi F, Ferro D, Quintarelli C, Saliola M, Cordova C, Balsano F. Clotting abnormalities in chronic liver disease. Dig Dis. 1992;10(3):162-72.

13.Francis JL, Armstrong DJ. Acquired dysfibrinogenaemia in liver disease. J Clin Pathol. 1982;35(6):667-72.

14. Martinez J, Palascak JE, Kwasniak D. Abnormal sialic acid content of the dysfibrinogenemia associated with liver disease. J Clin Invest. 1978;61(2):535-8.

15.Bashour FN, Teran JC, Mullen KD. Prevalence of peripheral blood cytopenias (hypersplenism) in patients with nonalcoholic chronic liver disease. Am J Gastroenterol. 2000;95(10):2936-9.
16. Peck-Radosavljevic M, Wichlas M, Zacherl J, Stiegler G, Stohlawetz P, Fuchsjager M, et al. Thrombopoietin induces rapid resolution of thrombocytopenia after orthotopic liver transplantation through increased platelet production. Blood. 2000;95(3):795-801.

17. Kajihara M, Kato S, Okazaki Y, Kawakami Y, Ishii H, Ikeda $Y$, et al. A role of autoantibody-mediated platelet destruction in thrombocytopenia in patients with cirrhosis. Hepatology. 2003;37(6):1267-76.

18. Kujovich JL. Hemostatic defects in end stage liver disease. Crit Care Clin. 2005;21(3):563-87.

19. Escolar G, Cases A, Vinas M, Pino M, Calls J, Cirera I, et al. Evaluation of acquired platelet dysfunctions in uremic and cirrhotic patients using the platelet function analyzer (PFA100 ): influence of hematocrit elevation. Haematologica. 1999;84(7):614-9.

20. Witters P, Freson K, Verslype C, Peerlinck K, Hoylaerts $\mathrm{M}$, Nevens F, et al. Review article: blood platelet number and function in chronic liver disease and cirrhosis. Aliment Pharmacol Ther. 2008;27(11):1017-29.

21. Goodpasture EW. Fibrinolysis in chronic hepatic insufficiency. Bull Johns Hopkins Hosp. 1914;25(285):330-6.

22. Biland L, Duckert F, Prisender S, Nyman D. Quantitative estimation of coagulation factors in liver disease. The diagnostic and prognostic value of factor XIII, factor $\mathrm{V}$ and plasminogen. Thromb Haemost. 1978;39(3):646-56.

23. Bakker CM, Metselaar HJ, Groenland TN, Gomes MJ, Knot EA, Hesselink EJ, et al. Increased tissue-type plasminogen activator activity in orthotopic but not heterotopic liver transplantation: the role of the anhepatic period. Hepatology. 1992;16(2):404-8.

24. Caldwell SH, Hoffman M, Lisman T, Macik BG, Northup PG, Reddy KR, et al. Coagulation disorders and hemostasis in liver disease: pathophysiology and critical assessment of current management. Hepatology. 2006;44(4):1039-46.

25. Papatheodoridis GV, Patch D, Webster GJ, Brooker J, Barnes E, Burroughs AK. Infection and hemostasis in decompensated cirrhosis: a prospective study using thrombelastography. Hepatology. 1999;29(4):1085-90.

26. Ben-Ari Z, Osman E, Hutton RA, Burroughs AK. Disseminated intravascular coagulation in liver cirrhosis: fact or fiction? Am J Gastroenterol. 1999;94(10):2977-82.

27. Joist JH. AICF and DIC in liver cirrhosis: expressions of a hypercoagulable state. Am J Gastroenterol. 1999;94(10):2801-3.

28. Agarwal S, Joyner KA, Jr., Swaim MW. Ascites fluid as a possible origin for hyperfibrinolysis in advanced liver disease. Am J Gastroenterol. 2000;95(11):3218-24.

29. Hou MC, Lin HC, Liu TT, Kuo BI, Lee FY, Chang FY, et al. Antibiotic prophylaxis after endoscopic therapy prevents rebleeding in acute variceal hemorrhage: a randomized trial. Hepatology. 2004;39(3):746-53. 
30. Thalheimer U, Triantos CK, Samonakis DN, Patch D, Burroughs AK. Infection, coagulation, and variceal bleeding in cirrhosis. Gut. 2005;54(4):556-63.

31. Montalto P, Vlachogiannakos J, Cox DJ, Pastacaldi S, Patch $\mathrm{D}$, Burroughs AK. Bacterial infection in cirrhosis impairs coagulation by a heparin effect: a prospective study. J Hepatol. 2002;37(4):463-70.

32. Noris M, Remuzzi G. Uremic bleeding: closing the circle after 30 years of controversies? Blood. 1999;94(8):2569-74.

33. Turitto VT, Baumgartner HR. Platelet interaction with subendothelium in a perfusion system: physical role of red blood cells. Microvasc Res. 1975;9(3):335-44.

34. Boccardo P, Remuzzi G, Galbusera M. Platelet dysfunction in renal failure. Semin Thromb Hemost. 2004;30(5):579-89.

35. Bellis L, Berzigotti A, Abraldes JG, Moitinho E, Garcia-Pagan JC, Bosch J, et al. Low doses of isosorbide mononitrate attenuate the postprandial increase in portal pressure in patients with cirrhosis. Hepatology. 2003;37(2):378-84.

36. Tripodi A, Salerno F, Chantarangkul V, Clerici M, Cazzaniga $M$, Primignani $M$, et al. Evidence of normal thrombin generation in cirrhosis despite abnormal conventional coagulation tests. Hepatology. 2005;41(3):553-8.

37. Starzl TE, Iwatsuki S, Van Thiel DH, Gartner JC, Zitelli BJ, Malatack JJ, et al. Evolution of liver transplantation. Hepatology. 1982;2(5):614-36.

38. Aldrete JA, Clapp HW, Starzl TE. Body temperature changes during organ transplantation. Anesth Analg. 1970;49(3):384-8.

39. Watts DD, Trask A, Soeken K, Perdue P, Dols S, Kaufmann C. Hypothermic coagulopathy in trauma: effect of varying levels of hypothermia on enzyme speed, platelet function, and fibrinolytic activity. J Trauma. 1998;44(5):846-54.

40. Doufas AG. Consequences of inadvertent perioperative hypothermia. Best practice \& research Clinical anaesthesiology. 2003;17(4):535-49.

41. Martini WZ. Fibrinogen metabolic responses to trauma. Scand J Trauma Resusc Emerg Med. 2009;17:2.

42. Lier H, Krep H, Schroeder S, Stuber F. Preconditions of hemostasis in trauma: a review. The influence of acidosis, hypocalcemia, anemia, and hypothermia on functional hemostasis in trauma. J Trauma. 2008;65(4):951-60.

43. Djaldetti $\mathrm{M}$, Fishman $\mathrm{P}$, Bessler $\mathrm{H}$, Chaimoff $\mathrm{C}$. $\mathrm{pH}$-induced platelet ultrastructural alterations. A possible mechanism for impaired platelet aggregation. Arch Surg. 1979;114(6):70710.

44. Engstrom M, Schott U, Romner B, Reinstrup P. Acidosis impairs the coagulation: A thromboelastographic study. J Trauma. 2006;61(3):624-8.

45. Hiippala ST, Myllyla GJ, Vahtera EM. Hemostatic factors and replacement of major blood loss with plasma-poor red cell concentrates. Anesth Analg. 1995;81(2):360-5.
46. Dzik WH. Component Therapy Before Bedside Procedures. In: Mintz PD, editor. Transfusion therapy: Clinical principles and practice. 2nd ed. Bethesda, MD: American Association of Blood Banks; 2005. p. 1-26.

47. Armand R, Hess JR. Treating coagulopathy in trauma patients. Transfus Med Rev. 2003;17(3):223-31.

48. Bohmig HJ. The coagulation disorder of orthotopic hepatic transplantation. Semin Thromb Hemost. 1977;4(1):57-82.

49. Dmitrewski J, Russell S, Vijeyasingham R, McMaster P. Hematologic problems and organ transplantation. World $\mathrm{J}$ Surg. 1996;20(9):1160-5.

50. Porte RJ, Knot EA, Bontempo FA. Hemostasis in liver transplantation. Gastroenterology. 1989;97(2):488-501.

51. Porte RJ, Bontempo FA, Knot EA, Lewis JH, Kang YG, Starzl TE. Systemic effects of tissue plasminogen activator-associated fibrinolysis and its relation to thrombin generation in orthotopic liver transplantation. Transplantation. 1989;47(6):978-84.

52. Kang YG, Martin DJ, Marquez J, Lewis JH, Bontempo FA, Shaw BW, Jr., et al. Intraoperative changes in blood coagulation and thrombelastographic monitoring in liver transplantation. Anesth Analg. 1985;64(9):888-96.

53. Porte RJ. Coagulation and fibrinolysis in orthotopic liver transplantation: current views and insights. Semin Thromb Hemost. 1993;19(3):191-6.

54. Homatas J, Wasantapruek S, Von Kaulla E, Von Kaulla KN, Eiseman B. Clotting abnormalities following orthotopic and heterotopic transplantation of marginally preserved pig livers. Acta Hepatosplenol. 1971;18(1):14-26.

55. Groth CG. Changes in coagulation. In: Starzl TE, Putman CW, editors. Experience in hepatic transplantation. Philadelphia: W.B. Saunders; 1969. p. 159-75.

56. Psuja P, Drouet L, Zawilska K. Binding of heparin to human endothelial cell monolayer and extracellular matrix in culture. Thromb Res. 1987;47(4):469-78.

57. Glimelius B, Busch C, Hook M. Binding of heparin on the surface of cultured human endothelial cells. Thromb Res. 1978;12(5):773-82.

58. Kettner SC, Gonano C, Seebach F, Sitzwohl C, Acimovic S, Stark J, et al. Endogenous heparin-like substances significantly impair coagulation in patients undergoing orthotopic liver transplantation. Anesth Analg. 1998;86(4):691-5.

59. Bakker CM, Stibbe J, Gomes MJ, Groenland TN, Metselaar $\mathrm{HJ}$, Hesselink EJ, et al. The appearance of donor heparin in the recipient after reperfusion of a liver graft. Transplantation. 1993;56(2):327-9.

60. Rehm M, Bruegger D, Christ F, Conzen P, Thiel M, Jacob $\mathrm{M}$, et al. Shedding of the endothelial glycocalyx in patients undergoing major vascular surgery with global and regional ischemia. Circulation. 2007;116(17):1896-906. 